\title{
A PROPOSED THROUGH-FLOW INVERSE METHOD FOR THE DESIGN OF MIXED-FLOW PUMPS
}

\section{ABSTRACT}

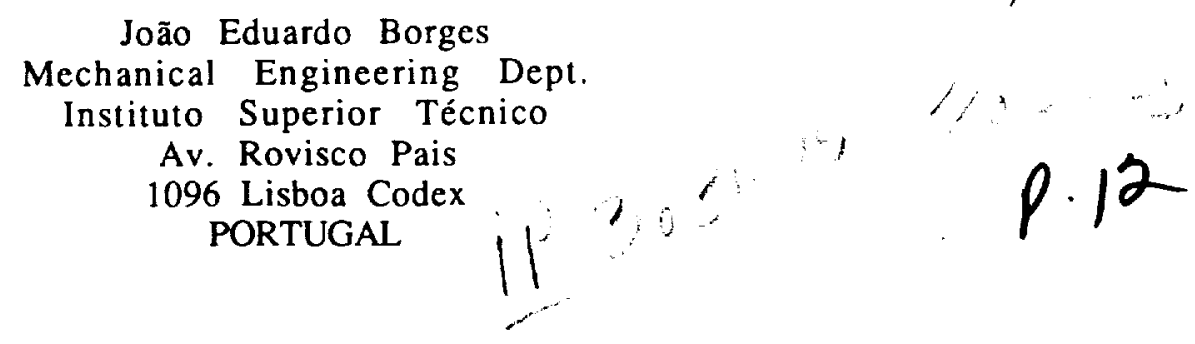

A through-flow (hub-to-shroud) truly inverse method is proposed and described in this paper. It uses as a design initial specification an imposition of mean swirl, i.e., radius times mean tangential velocity, given throughout the meridional section of the turbomachine. In the present implementation, it is assumed that the fluid is inviscid, incompressible and irrotational at inlet and the blades are supposed to have zero thickness. Only blade rows that impart to the fluid a constant work along the span will be considered.

An application of this procedure to design the rotor of a mixed-flow pump will be described in detail. The strategy used to find a suitable mean swirl distribution and the other design inputs is also described. The final blade shape and pressure distributions on the blade surface are presented, showing that it is possible to obtain feasible designs using this technique. Another advantage of this technique is the fact that it does not require large amounts of CPU time.

\section{1-INTRODUCTION AND LITERATURE SURVEY}

The large majority of pumps is designed by using very simple and rudimentary one-dimensional considerations concerning the velocity triangles, considerations which allow the calculation of the evolution of the blade angle along the passage, see for example [1] and [2]. These methods are so easy that they can be carried out using only hand calculations and simple graphical processes.

Although some pumps are still being calculated using hand calculations, the above one-dimensional procedure can and has been programmed as computer codes which are being used by the most important pump manufacturers. The results obtained with these techniques are the better the more radial the blade passage is. However, for mixed-flow pumps, the velocity triangles vary appreciably along the span, so that the above methodology is not good enough. In order to take into account this effect in some way, designers usually split the flow passage in several parts, applying the above considerations to each one.

When the pump designs involve some responsibility, the above step is followed by a verification using a direct code which is run with the geometry arrived at previously. Before reaching the final design, several iterations following the above steps are usually required.

This complete process can be time consuming, so that one is left wondering whether it could be improved. One possible way to achieve this could be by using inverse methods, enabling the achievement of the blade row layout in a more direct form, in one single step. Among inverse methods, two-dimensional techniques are the most frequently used and the ones that require less CPU time. These two-dimensional inverse methods can be classified into two main groups, according to the approximations used when looking for the solution to the blade design. The first sort of approximations gives rise to blade-to-blade methods since the calculations are done in the blade-to-blade plane. This kind of methods is popular among designers of axial turbomachinery, but it did not attract much attention among designers of radial

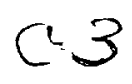


turbomachinery. The reason may be connected to the fact that the flow passages are more complicated and there are significant changes in radius.

The other way of tackling the solution consists in working in the hub-toshroud plane (i.e., in the meridional plane), giving rise to what we could call hub-toshroud (or through-flow) methods. In this sort of approach the flow is supposed axisymmetric, an approximation that can be interpreted as giving the mean flow through the turbomachine. This sort of approach seems more popular among radial turbomachinery designers. In fact, one can find examples of this kind of procedure as far back as 1955, when reference [3] described some work applied to the redesign of a centrifugal compressor, using a hub-to-shroud method. In this instance the blade shape was kept fixed and given as input, while the shroud contour was altered and evolved as a result of the calculations. The centrifugal compressors described in [3] were built and tested, the experimental results being presented in [4]. The experimental results show that this technique produced significant improvements in the overall efficiency and peak pressure ratio.

A different strategy was followed in reference [5] which, again, describes the application of an inverse technique to the design of centrifugal compressors. Contrary to the previous example the meridional geometry (hub and shroud contours) of the machine is supposed known and given as input. The other inputs consist of a suitable normal blade thickness and the desired velocity loading (difference in velocity across the blade) at hub, mid-span and shroud as a function of distance along the camberline. As a result of the calculations the blade shape was obtained. This paper presents some experimental evidence suggesting that the procedure gives reliable results when the flow is attached.

The method proposed in the present work has got some similarities to that of [5] in the sense that it is also a hub-to-shroud inverse technique that assumes as known the meridional geometry and calculates the blade shape that will satisfy some flowfield conditions, given as input to the procedure. The input design specification used

here is a mean swirl (radius times mean tangential velocity, $\mathrm{r} \overline{\mathrm{V}}_{\theta}$ ) distribution given throughout the meridional section. This design specification is somewhat unusual, but a suggestion in this direction can already be found in the work of $W u$ (see [6]) and it is ideally suited to the design of radial turbomachinery as discussed in [7]. In fact, the work of [7], which presents a three-dimensional inverse method using a mean swirl specification, shows that the mean swirl specification is related to the way the work is imparted to the fluid as it passes through the blade row. In other words, the mean swirl can be related to the blade loading across the blades. Another reference that discusses the use of a mean swirl imposition is [8], where the equations to be used in the present work are derived. Nevertheless [8] does not present any practical examples of application of the equations.

\section{2-DESCRIPTION OF THE DESIGN METHOD}

Throughout this work we will use a right-handed cylindrical polar coordinate system defined by $(r, \theta, z)$, where $r$ is the radius, $\theta$ the angular coordinate and $z$ is the axial distance. In addition, we will use an auxiliary coordinate $\alpha$, defined by:

$$
\alpha=\theta-f(r, z)
$$

where $f(r, z)$ is the angular coordinate of a point on the blade camber surface. This variable $\alpha$ can be interpreted as a sort of helical angular coordinate aligned with the blade, so that when

$$
\alpha=m \frac{2 \pi}{B}
$$


with $\mathrm{m}$ an integer $(\mathrm{m}=\ldots,-1,0,1,2,3, \ldots)$ and $\mathrm{B}$ equal to the number of blades of the turbomachine, we are on a blade surface (eq. (2) describes the blade shape).

In the following we will use bold underlined characters for vectors and mean values will be denoted with an overbar.

\subsection{Velocity Flow Field.}

Since it was intended to apply this method to design pumps, it was assumed that the fluid was inviscid and incompressible and, for simplicity sake, the blade thickness is not considered in this procedure.

In agreement with the through-flow approximation, the flow through the turbomachine will be assumed axisymmetric even in the blade region. This mean velocity field will be calculated using the streamfunction concept and the value of the mean vorticity. Indeed, since the vorticity field is solenoidal, it can be written as the cross product of two gradients of scalar functions. One of these scalar functions may be $\alpha$ according to the fact that all the vorticity is confined to the blades. In fact, if we suppose the far upstream velocity is uniform (an approximation quite frequent), we can say the flow is irrotational at inlet. Concentrating in designs that execute constant work along the span, it is concluded that the flow must remain everywhere irrotational according to Kelvin's theorem. So, if there is any vorticity at all, it must be bound to the blade surfaces, justifying the statement just made. The other scalar function in the expression for the vorticity turns out to be the mean swirl as is shown in [7]. Therefore, the expression for the mean vorticity, $\bar{\Omega}$ is:

$$
\overline{\mathbf{\Omega}}=\mathbf{r} \overline{\mathrm{V}}_{\boldsymbol{\theta}} \times \nabla \alpha
$$

and now that the mean vorticity is known, the corresponding velocity field can easily be calculated. It is indeed known that the mean vorticity is the curl of the mean velocity $\overline{\mathbf{Y}}$, or:

$$
\overline{\mathbf{\Omega}}=\nabla \times \overline{\mathbf{Y}}
$$
obtained:

Equating the $\theta$-component of eqs. (3) and (4) the following equation is

$$
\frac{\partial \bar{V}_{r}}{\partial z}-\frac{\partial \bar{V}_{z}}{\partial r}=\frac{\partial f}{\partial z} \frac{\partial r \bar{V}_{\theta}}{\partial r}-\frac{\partial f}{\partial r} \frac{\partial r \bar{V}_{\theta}}{\partial z}
$$

relating the velocity field to the blade shape, $f$, and the mean swirl $r \bar{V}_{\theta}$. Besides this equation, the velocity field must satisfy the continuity equation. In order to achieve this we introduce the concept of a streamfunction defined by:

$$
\begin{aligned}
& \bar{V}_{r}=-\frac{1}{r} \frac{\partial \psi}{\partial z} \\
& \bar{V}_{z}=\frac{1}{r} \frac{\partial \psi}{\partial r}
\end{aligned}
$$



ICIDES-III) Edivor: G.S. Dulikravich. Washingion D.Cu Osbober 23-25. 1991.

so that this definition satisfies identically the continuity equation for incompressible

flow, i. e., $\nabla \cdot \overline{\mathbf{y}}=0$. The actual value of $\psi$ appearing in the definitions (6a) and (6b) is going to be determined substituting (6a) and (6b) in eq. (5). In this way we arrive at:

$$
\frac{\partial^{2} \psi}{\partial \mathrm{r}^{2}}-\frac{1}{\mathrm{r}} \frac{\partial \psi}{\partial \mathrm{r}}+\frac{\partial^{2} \psi}{\partial \mathrm{z}^{2}}=-\mathrm{r}\left(\frac{\partial \mathrm{f}}{\partial \mathrm{z}} \frac{\partial \mathrm{r} \bar{V}_{\theta}}{\partial \mathrm{r}}-\frac{\partial \mathrm{f}}{\partial \mathrm{r}} \frac{\partial \mathrm{r} \bar{V}_{\theta}}{\partial \mathrm{z}}\right)
$$

For the resolution of this equation it is necessary to specify a complete set of boundary conditions. The boundary condition to use along the endwalls (hub and shroud) is the one that states that there is no flow through the solid walls. Using the streamfunction concept, this fact is expressed as:

$$
\psi=\text { Const. }
$$

or, in other words, the hub and shroud must be streamlines of the flow.

Far upstream we know the mean velocity vector, $\overline{\mathrm{V}}_{-\infty}$, since it is given as input. Therefore we can write:

$$
\frac{1}{\mathbf{r}} \frac{\partial \Psi}{\partial \mathbf{s}}=\overline{\mathbf{V}}_{-\infty} \cdot \mathbf{n}
$$

where $\mathbf{s}$ is distance along the far upstream boundary and $\mathbf{n}$ is the unitary vector perpendicular to it. This expression enables us to calculate the values of $\psi$ along the far upstream boundary, using a simple numerical integration. At the far downstream boundary a similar expression applies since the velocity there is uniform because the flow is irrotational at inlet and the blade row is supposed to execute constant work along the span. In this way the complete set of boundary conditions is obtained.

The partial differential equation (7) was solved using finite difference techniques. As a typical mixed-flow pump has a meridional section with complicated geometry bounded by curved boundaries (hub and shroud profiles) it was decided to use a transformation of coordinates to body-fitted curvilinear coordinates (see [9]). Since this coordinate system should be easy to generate and require little computational time, it was decided to use an algebraic transformation. For this kind of transformation of coordinates, mesh points are distributed along quasi-orthogonals and quasi- streamlines. Fig. 2 shows the grid used in the calculations to be discussed later on.

Eq. (7) was discretized using second-order accurate central difference formulae, obtaining a nine-point difference star. The resulting finite difference equations were solved by a relaxation method. In the present case, a Gauss-Seidel relaxation scheme was used, implemented in conjunction with a multi-grid technique in order to accelerate the convergence rate of the solution. A good description of multi-grid methods can be found in [10], and, in fact, the relaxation subroutines used in our program are a slightly modified version of the ones presented in [10].

\subsection{Equation for the Determination of the Blade Shape.}

After calculating the velocity field using the information presented in the previous subsection, it is necessary to evaluate the blade geometry. That is done by requiring the blade to be tangent to the velocity vector. This condition can be expressed as:

$\overline{\mathbf{W}} \cdot \nabla \alpha=0$ 
where $\overline{\mathbf{W}}$ is the local relative velocity, $\overline{\mathbf{W}}=\overline{\mathbf{V}}-\underline{U}$. Expanding eq. (10), the following expression is obtained:

$$
\bar{V}_{z} \frac{\partial f}{\partial z}+\bar{V}_{r} \frac{\partial f}{\partial r}=\frac{r \bar{V}_{\theta}}{r^{2}}-\omega
$$

where $f$ is the angular coordinate of the blade and $\omega$ is the rotational speed of the blade row. $\bar{V}_{z}$ and $\bar{V}_{r}$ are the mean axial and radial velocities, respectively.

Eq. (11) is a first-order partial differential equation with characteristic lines coincident with the meridional projection of the streamlines. In order to integrate this differential equation, some initial data must be specified along a line roughly perpendicular to these characteristic lines and extending from hub to shroud. This initial data on $f$ will be called the stacking condition of the blade. In our method this stacking condition is implemented by giving, as input, the values of the blade coordinate $f$, along a quasi-orthogonal, for example, at the leading edge.

After the stacking condition is specified, we can integrate eq. (11), since all the velocities that appear in it are known from the previous iteration. The integration of eq. (11) was done using finite difference methods. More specifically, an Euler's modified method (see [11]) was used. This is an implicit numerical scheme that has a truncation error of second order in the mesh size and is consistent and stable.

\subsection{Estimation of Blade Surface Pressures.}

One result that is important in any design method is the values of pressure on the suction and pressure surfaces of the blade, for judging whether the pressure distribution is adequate or not. In order to estimate these values starting from the axisymmetric solution it is necessary to calculate the blade surface velocities using the mean values known. To do that we begin by determining the velocity jump across the blades $\left(\underline{\mathbf{W}}^{+}-\underline{\mathbf{w}}^{-}\right)$which is given by (see [7]):

$$
\underline{\mathbf{w}}^{+}-\underline{\mathbf{w}}^{-}=\frac{2 \pi}{\mathrm{B}} \frac{\left(\nabla \mathrm{r} \overline{\mathrm{V}}_{\theta} \times \nabla \alpha\right) \times \nabla \alpha}{\nabla \alpha \cdot \nabla \alpha}
$$

where, for a pump, $\underline{W}^{+}$is the relative velocity at the pressure surface and $\mathbf{W}^{-}$is the relative velocity at the suction surface. This expression is physically plausible as it gives a jump in velocity which lies on the blade since it is normal to $\nabla a$ (a vector itself normal to the blade). In addition, we would expect the jump to be normal to the

vorticity vector, $\frac{2 \pi}{B}\left[\nabla r \bar{V}_{\theta} \times \nabla \propto\right]$, lying in the blade. Knowing the velocity jump and assuming the velocity profile is linear between suction and pressure surfaces (a frequent approximation in hub-to-shroud methods, see [12]) it is possible to estimate the velocities at the suction and pressure surfaces. Indeed, their values are going to be equal to the mean velocity (solution of the axisymmetric problem) plus or minus onehalf the velocity jump. Afterwards, using the fact that the flow is irrotational at inlet and applying Bernoulli's equation, the expression for the difference in pressure across the blades is obtained. The final expression is (see [7]):

$$
\mathrm{p}^{+}-\mathrm{p}^{-}=\frac{2 \pi}{\mathrm{B}} \rho \overline{\mathbf{W}} \cdot \nabla \mathrm{r} \overline{\mathrm{V}}_{\theta}
$$


where $\mathrm{p}^{+}$is the pressure at the pressure surface, $\mathrm{p}^{-}$the pressure at the suction

surface and $\rho$ is the fluid density. $\overline{\mathbf{W}}$ is known from the velocity field calculation so that all the terms in the right-hand side of eq. (13) are known, enabling the estimation of the pressure loading across the blades.

\subsection{Flowchart.}

To end section 2, we would like to draw the attention of the reader to the fact that the vorticity depends on the blade shape, $f$, which in its tum is an outcome of the calculations. So, the calculations must be iterated until convergence is obtained. This is done according to the following flowchart:

(i) input of initial data - specified values of $r \overline{\mathrm{V}}_{\theta}$, definition of meridional section and body-fitted curvilinear coordinate system and all the relevant parameters;

(ii) estimation of a first guess for $f$, assuming that the mean velocity is uniform along the quasi-orthogonals;

(iii) using the input values of mean swirl, $\mathrm{r} \overline{\mathrm{V}}_{\theta}$, and the values of blade shape, $f$, from last iteration, calculate the mean velocity field (solution of eq. (7));

(iv) update the blade shape, $f$, by integration of eq. (11);

(v) if the solution is converged output the blade shape, $f$, and other relevant results. Otherwise go back to step (iii) and initiate a new iteration.

This flowchart was implemented as a FORTRAN computer code and applied to the design of the impeller of a mixed-flow pump.

\section{3- DISCUSSION OF APPLICATION TO A MIXED-FLOW PUMP}

In order to show the potentialities of the method, it was decided to apply it to the design of a mixed-flow pump. The chosen pump was based on a real machine which had as nominal conditions a value of $28 \mathrm{~m}$ for the head, $H$, a nominal volume flow, $Q$, of $600 \mathrm{~m}^{3} / \mathrm{h}$ and a rotational speed of 1450 r.p.m.. These values give a nondimensional specific speed parameter equal to 0.919 . The rotor has 8 blades and a tip diameter equal to $320 \mathrm{~mm}$. This value will be used to non-dimensionalize all the linear dimensions and the velocities will be made nondimensional by using the transport blade tip velocity, $\omega \mathrm{r}_{\text {tip }}$ (its value is $24.3 \mathrm{~m} / \mathrm{s}$ ).

The meridional geometry used in the calculations is based on an existing pump (with minor alterations), designed by a Portuguese pump manufacturer using hand calculations and graphical processes. The final meridional shape used is defined in Fig. 1. A grid formed by 145 quasi-orthogonals and 57 quasi-streamlines was fitted to this meridional section, there being in the blade region a total of $61 \times 57$ points. Fig. 2 shows every other line of the grid used. As can be seen, a region upstream and downstream of the blade zone was considered in the calculations.

An important input to the present inverse method is the specification of mean

swirl, $\mathrm{r} \overline{\mathrm{V}}_{\theta}$. As it is supposed that the pump accepts the flow with no swirl, the value of

$\mathrm{r} \overline{\mathrm{V}}_{\boldsymbol{\theta}}$ along the entire leading edge is considered equal to zero. At the trailing edge the value was also considered constant in order to obtain a design that executes constant

work along the span. The necessary value of $\mathrm{r} \overline{\mathrm{V}}_{\theta}$ at the trailing edge depends on the work per unit mass of fluid desired for the rotor, which is a value determined by the desired head and an assumed value of efficiency (in our case considered equal to 0.86).

The value of $r \bar{V}_{\theta}$ used at the trailing edge is $0.5411 \omega \mathrm{r}_{\text {tip }}^{2}$. 
Another restriction was imposed on the derivatives of the mean swirl at the leading and trailing edges, namely, we forced there a zero derivative along the quasistreamlines. This was done in order to obtain a zero pressure loading at the trailing edge (as is required by the Kutta-Joukowski condition), and at the leading edge. The equivalence between a zero derivative of the mean swirl and zero pressure loading can be seen from expression (13) which shows that the pressure loading depends on

the gradient of mean swirl, being zero where the gradient of $r \bar{V}_{\theta}$ is zero, as it is the case at the leading and trailing edges.

This close equivalence between derivatives of mean swirl and pressure loading across the blade was one of the factors used when choosing the input mean swirl, $\mathrm{rV}_{\boldsymbol{\theta}}$, along the entire meridional section, and whose contours are presented in Fig. 3 . The other factor considered was the attempt to avoid a blade shape too twisted which would be difficult to manufacture. These two factors were exactly the same guidelines advanced and discussed in [7] when choosing the mean swirl for a completely different turbomachine, a radial inflow turbine.

Recalling briefly the arguments advanced in [7] and which are sufficiently general to apply to the present situation, it is evident from expression (13) that the pressure blade loading is proportional to the product of the modulus of the relative velocity and the value of the derivative of $\mathrm{r}_{\theta}$ along the meridional projection of the flow streamlines, or:

$$
\mathrm{p}^{+}-\mathrm{p}^{-}=\frac{2 \pi}{\mathrm{B}} \rho|\overline{\mathbf{W}}| \frac{\partial \mathrm{r} \overline{\mathrm{V}}_{\theta}}{\partial \mathrm{s}}
$$

where $s$ is distance along the meridional projection of the streamlines. In a well designed machine, $|\overline{\mathbf{W}}|$ does not vary abruptly and the streamlines have a direction close to the quasi-streamlines. Therefore, eq. (14) implies that the pressure blade loading is mainly influenced by the value of the derivative of $r \bar{v}_{\theta}$ along the quasistreamlines, which is a value known at the start of the calculations, and so can be controlled. In this way, if it is desired to design a blade with a big loading near the leading edge, then the derivatives of $\mathrm{r} \bar{V}_{\theta}$ along the quasi-streamlines should have large values near the leading edge. In addition, if one wishes to obtain a pressure loading with a smooth evolution, then the derivatives of $\mathrm{r}_{\theta}$ should be watched with special care, specifying them as smooth as possible and with a monotonic variation from the leading to the trailing edge. From the point of view of the pressure loading the most unfavourable situation is along the shroud, so that there the derivatives should have a smooth variation.

The other argument that one should bear in mind when choosing the input mean swirl schedule is the one connected with the amount of blade twist. In order to clearly understand this argument it is important to rewrite eq. (11) along a streamline, obtaining: 

ICIDES-III) Editor: G.S. Dulikravich. Washingion D.C. Ockober 23-25. 1921.

$$
f_{a}-f_{b}=\int_{b}^{a} \frac{\bar{w}_{\theta}}{r \bar{V}_{m}} d m=\int_{b}^{a} \frac{\left(\bar{V}_{\theta}-\omega r\right)}{r \bar{V}_{m}} d m
$$

where $a$ and $b$ are two arbitrary points on the same streamline, $f$ is the angular coordinate of the blade, $\overline{\mathrm{V}}_{m}$ is the mean velocity in the meridional plane and $\mathrm{m}$ is the distance along the meridional projection of the streamline. If one wishes to control the total variation in the angular coordinate of the blade, $f_{a}-f_{b}$, it is convenient to avoid high values for the expression under the integral sign in eq. (15). This can be achieved if one specifies the value of $r \bar{V}_{\theta}$ so that $\bar{W}_{\theta}$ has small values, or in other words, if one specifies $r \overline{\mathrm{V}}_{\theta}$ so that the value of $\overline{\mathrm{V}}_{\theta}$ closely follows the local value of the transport velocity, $\omega \mathrm{r}$. This is the more important, the lower is the value of radius and

of the meridional velocity $\bar{V}_{m}$. From the point of view of highly twisted blades, the most critical streamsurface is the hub, not only because there the radius and meridional velocity take the lower values in the machine, but also because the meridional flow path is usually longer along the hub than anywhere else in the machine.

The next set of four figures is presented to demonstrate that the two above points were taken into consideration. Indeed, Fig. 4 gives the evolution of the input $r \bar{V}_{\theta}$ on the hub and the shroud. It is clearly seen that on the shroud the evolution of $r \bar{V}_{\theta}$ is quite gradual and smooth while at the hub the opposite happens.

Fig. 5 shows, on the same graph, the values of $\omega r$ and the specified values of $\bar{V}_{\theta}$ at the hub. Here it is evident that, on the hub, $\bar{V}_{\theta}$ has an evolution which is almost parallel to $\omega r$, for most of the flow path and without much consideration in the direction of obtaining smooth derivatives. This was done so that $\overline{\mathrm{W}}_{\theta}$ at the hub presented small and approximately constant values along most of the blade, leading to a reasonable overall change in the values of blade angular coordinate, $f$.

From the next figure, Fig. 6, it can be seen that the contrary happens on the

shroud, where $\bar{V}_{\theta}$ was chosen to have a smooth variation rather than following the local value of blade speed, $\omega r$. In fact, the main concern when specifying the mean swirl at the shroud was to obtain an adequate pressure distribution and not to control the overall variation in the blade angular coordinate, $f$.

The above ideas are corroborated by the next figure, Fig. 7 , where the

derivatives of $r \bar{V}_{\theta}$ along the quasi-streamlines for the hub and the shroud are presented. As can be clearly seen, the derivatives at the shroud present a smooth variation while the same does not apply at the hub, where a more abrupt change of the $r \bar{V}_{\theta}$ derivatives can be detected.

Using the close relationship between $r \bar{V}_{\theta}$ derivatives and pressure loading one can conclude that the present design presents a large loading near the leading edge at 
the shroud streamsurface, while at the hub streamsurface the blade loading is more evenly spread. However, one should point out that, at the hub, the derivative is zero for approximately the last $15 \%$ of the meridional flow path. This indicates that there will be a small pressure loading along the last portion of the blade at the hub, suggesting that perhaps one could use a shorter flow path there. This idea would entail an alteration of the trailing edge shape (using a trailing edge inclined to the axis, instead of parallel) and so, was not pursued further.

The inverse computer code was run, using the input described above together with a stacking condition imposed at the trailing edge and which consists of a linear variation of $\mathrm{f}$ between the value of 0.0 (at the hub) and 0.10 (at the shroud). As a result the blade shape described in the next figure was obtained. Since it is difficult to visualize the blade three-dimensional geometry, we decided to present the blade geometry in Fig. 8 as a view of two consecutive blades, as would be seen by an observer looking in the direction of the impeller axis. The blade obtained seems typical of a pump impeller and no particular problems are envisaged during its manufacture, since it is not a highly twisted blade.

In the next plot. Fig. 9, it is presented the estimated pressure distribution on hub and shroud, assuming a linear variation of the velocity from suction to pressure surfaces and using the procedure already discussed in subsection 2.3. The pressure coefficient, $C_{p}$, used in this plot is defined as:

$$
C_{p}=\left(\frac{\underline{w}}{\underline{\underline{y}}_{\mathrm{ref}}}\right)^{2}-1
$$

where $\underline{W}_{r e f}$ is a reference relative velocity, which is equal to $0.311 \omega \mathrm{r}_{\text {tip }}$ in the present case. Notice that the loading has a behaviour quite similar to the evolution of the derivatives of $\mathrm{r}_{\theta}$ along the quasi-streamlines, shown in Fig. 7 , bearing out the comments made above, concerning the close relationship between derivatives of $r \bar{V}_{\theta}$ and pressure loading. For example, it is seen that, at the hub, the pressure loading varies more abruptly than at the shroud, and along the last $15 \%$ of the flow path at hub the pressure loading is zero as was already expected from the values of derivatives of mean swirl. It should also be remarked that the distribution of pressure on the blade surfaces at the hub is not ideal since its variation is not smooth and presents some decelerations. However, the optimization of the pressure distribution would entail changes in the meridional section of the machine, and so was not tried in this work.

One advantage of the present method lies in the fact that it is quite rapid, requiring small amounts of CPU time. In fact, the present run required $1 \mathrm{~m} 39 \mathrm{~s}$ of CPU time in a VAX 3400 computer. Since it is computationaly so cheap, several different input mean swirl distributions can be scanned quickly, enabling the choice of the most appropriate mean swirl schedule.

\section{CONCLUSLONS}

A through-flow (hub-to-shroud) inverse method was proposed and implemented as a computer code. In the present implementation the flow is assumed incompressible, irrotational at inlet and the blade thickness was not considered during the calculations. The necessary equations are presented and, as an example of a possible application, the inverse method was used to redesign the rotor of a mixed-flow pump.

The present method uses as an input specification the value of mean swirl,

$\mathbf{r} \bar{V}_{\theta}$. This input specification was chosen using a reasoning similar to that used in [7]. This is remarkable, since the turbomachine designed in [7] was a radial-inflow 
turbine, and indicates that the strategy developed there is quite general, being able to cope with radically different machines. Briefly, this strategy consists in using the input mean swirl to control the pressure loading on the blades and the overall variation in the angular coordinate of the blade, $f$.

This work also shows that further research should be done in order to obtain reasonable pressure distributions on the blade surfaces. In fact, the design presented here has a pressure distribution on the hub which is not the ideal one. The optimization of the pressure distribution will probably involve some changes in the meridional section (hub and shroud contours).

One advantage of the present technique is the fact that it requires small amounts of CPU time. So it is a convenient tool to scan quickly and inexpensively several different input mean swirl distributions, in order to find the most appropriate one.

\section{REEERENCES}

[1] Lazarkiewicz, S. and Troskolanski, A.T., Impeller Pumps, Pergamon Press, Oxford, 1965.

[2] Lobanoff, V. S. and Ross, R. R., Centrifugal Pumps: Design \& Application, Gulf Publishing Company, Houston, Texas, chapter 3, pp. 28-45, 1985.

[3] Smith, K. J. and Hamrick, J. T., A Rapid Approximate Method for the Design of Hub Shroud Profiles of Centrifugal Impellers of Given Blade Shape, NACA Technical Note 3399, 1955.

[4] Osbom, W. M.; Smith K. J. and Hamrick, J. T. , Design and Test of Mixed-Flow Impellers VIII- Comparison of Experimental Results for Three Impellers with Shroud Redesigned by Rapid Approximate Method, NACA Research Memorandum E56L07, 1957.

[5] Jansen, W, and Kirschner, A. M., Impeller Blade Design Method for Centrifugal Compressors, NASA SP304, Pt. 2, pp. 537-563, 1974.

[6] Wu, C.-H., A General Theory of Three- Dimensional Flow in Subsonic and Supersonic Turbomachines of Axial-, Radial- and Mixed-Flow Types, NACA Technical Note 2604, January 1952.

[7] Borges, J. E., A Three-Dimensional Inverse Method for Turbomachinery: Part ITheory, Transactions of the ASME, Journal of Turbomachinery, Vol. 112, pp. 346-354, 1990.

[8] Hawthorne, W. R., The Actuator Duct Representation of Turbomachinery Blade Rows, CUED/A- Turbo/TR 119, 1983.

[9] Thompson, J. F.; Warsi, Z. U. A. and Mastin, C. W., Boundary-Fitted Curvilinear Coordinate Systems for Solution of Partial Differential Equations - A Review, Journal of Computational Physics, $\mathrm{n}^{9} 47$, pp. 1- 108, 1982.

[10] Brandt, A., Multi-Level Adaptive Solutions to Boundary-Value Problems, Mathematics of Computation, Vol. 31, $\mathrm{n}^{\mathrm{9}}$ 138, pp. 333-390, 1977.

[11] Roache, P. J., Computational Fluid Dynamics, Hermosa Publishers, Albuquerque, N.M., U.S.A., pp. 84-85, 1982.

[12] Stanitz, J. D. and Prian, V. D., A Rapid Approximate Method for Determining Velocity Distribution on Impeller Blades of Centrifugal Compressors, NACA Technical ' Note 2421, 1951. 
Third Intemational Conference on Inverse Design Concepts and Optimization in Engineering Sciences (ICIDES-III) Edivor: C.S. Dulikravich. Washinguen D.C. October 23-25.1991

ACKNOWLEDGEMENTS

The work described here was financially supported by CTAMFUTL/INIC. The suggestions and help of the technical staff of EFACEC are also gratefully acknowledged.

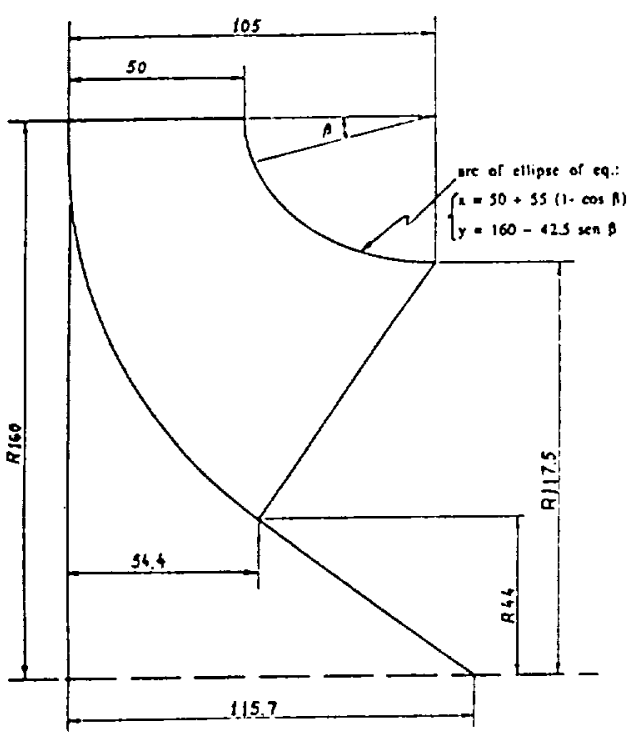

Fig. 1 Definition of meridional geomelry

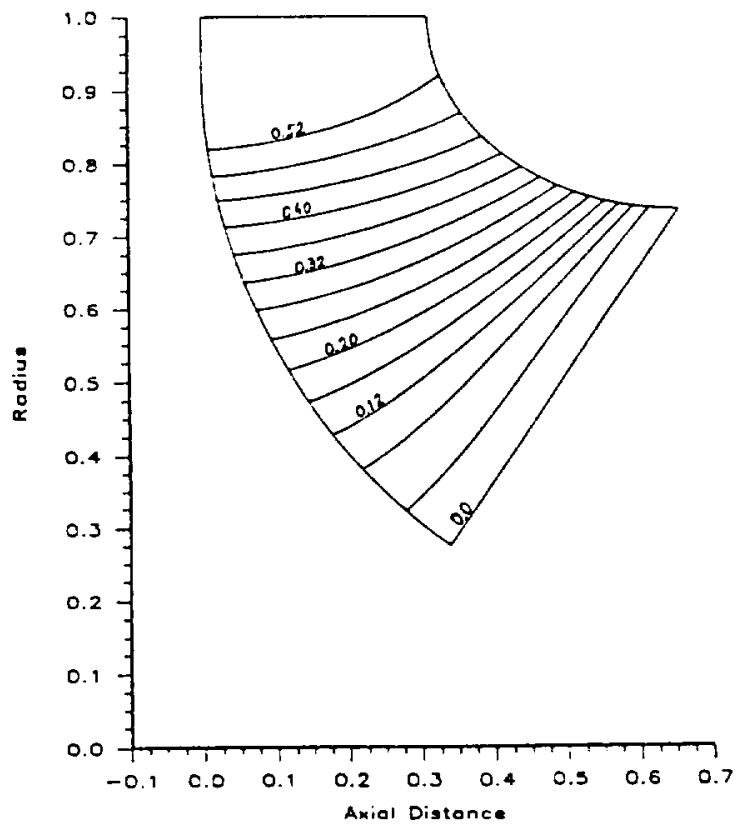

Fig. 3 Contours of the input mean swirl distribution (defined as $r \bar{v}_{\theta} /$ (tip $U_{\text {tip }}$ )

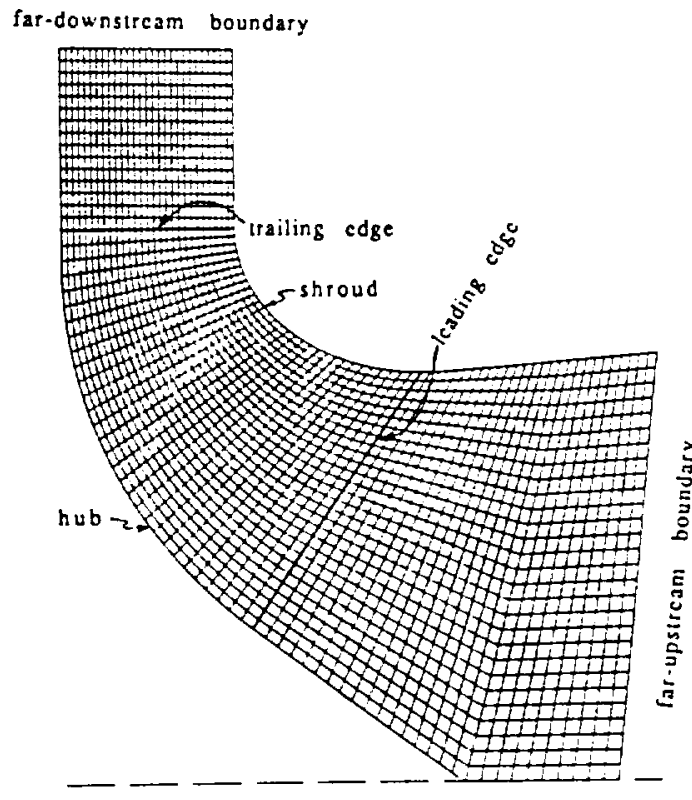

Fig. 2 Grid used in the meridional plane

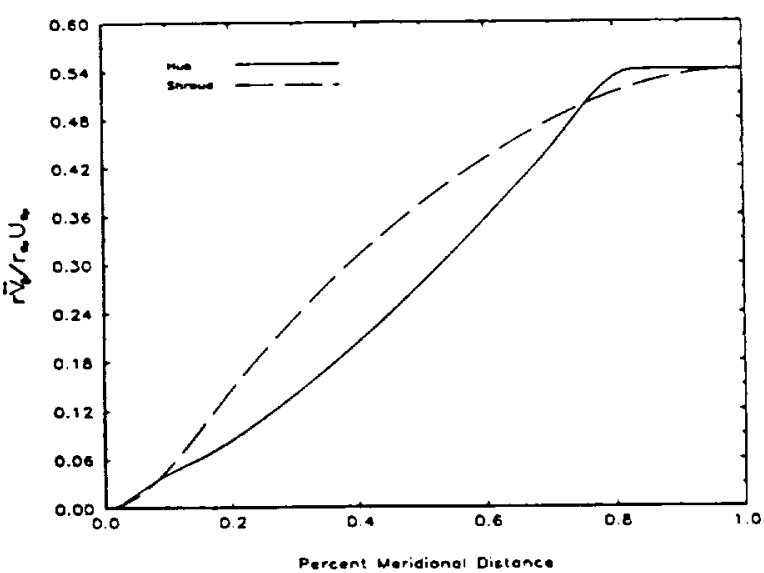

Fig. 4 Input mean swirl distribution at hub and shroud 
Third International Conference on Inverse Design Concepts and Opumization in Engineering Sciences ICIDES-III) Edivor. C.S. Dulikravich. Washinglon D.Cu Octeber 23-25.1991.

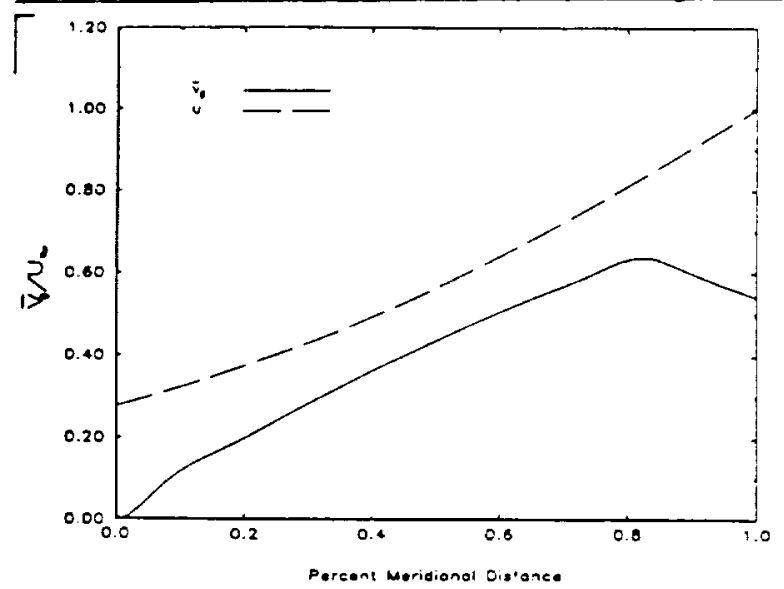

Fig. 5 Mean tangential velocily and blade speed at hub

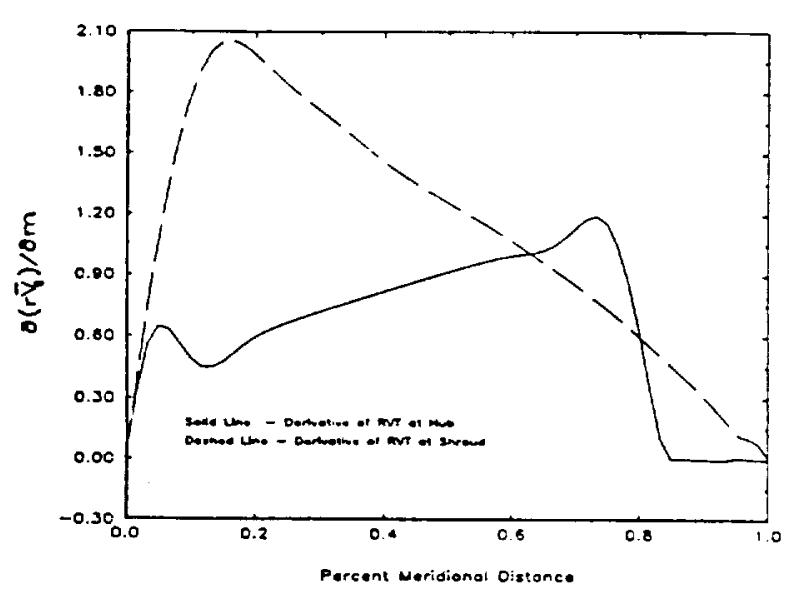

Fig. 7 Derivatives of the input mean swirl distribution

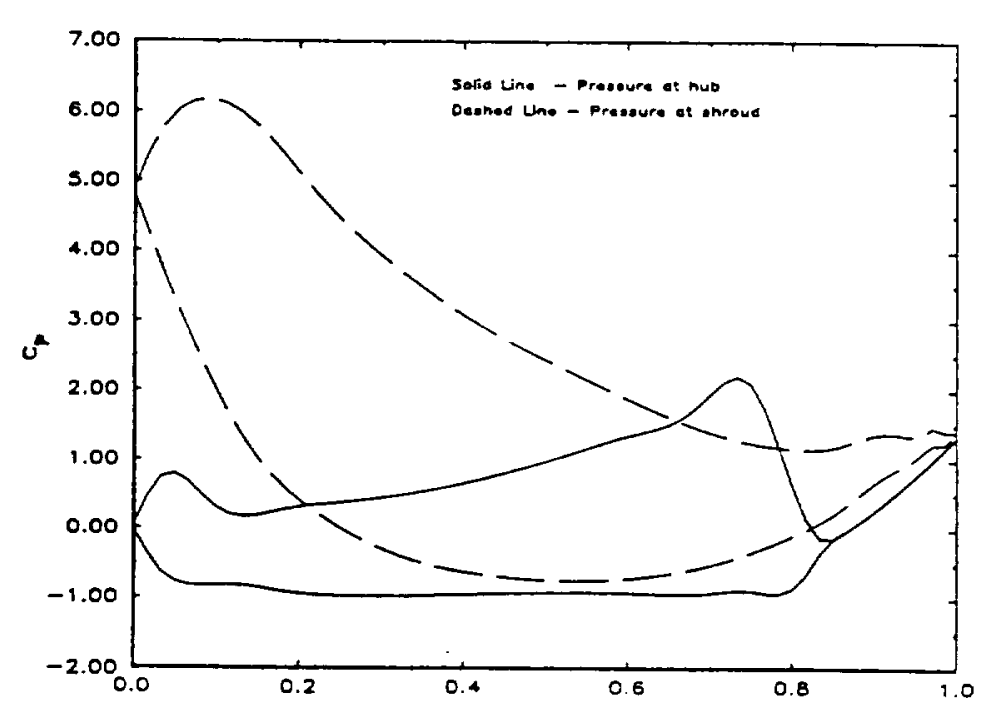

Percent Meridional Distance

Fig. 9 Pressure disiribution on hub and shroud

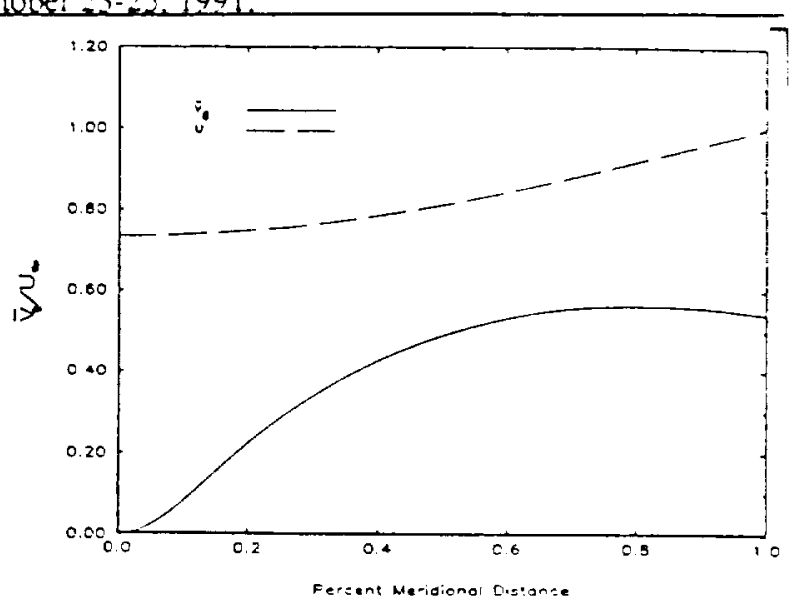

Fig. 6 Mean tangential velocity and blade speed at shroud

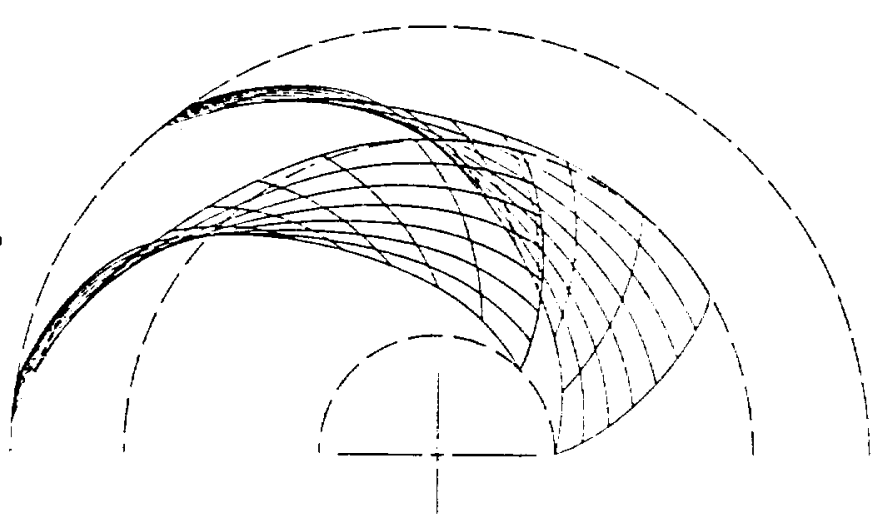

Fig. 8 Blade projection along the axis 